\title{
Insight into the Structural Variations of Fergusonite-Type Structures: Combined Experimental and Computational Studies
}

\author{
B. G. Mullens ${ }^{1}$, M. Avdeev ${ }^{1,2}$, H. E. A. Brand ${ }^{3}$, S. Mondal', G. Vaitheeswaran ${ }^{5}$, and B. J. Kennedy ${ }^{1}$ \\ ${ }^{1}$ School of Chemistry, The University of Sydney, Sydney, New South Wales 2006, Australia, \\ ${ }^{2}$ Australian Nuclear Science and Technology Organisation, Lucas Heights, New South Wales 2234, Australia, \\ ${ }^{3}$ Australian Synchrotron, 800 Blackburn Road, Clayton, Victoria 3168, Australia, \\ ${ }^{4}$ Advanced Centre of Research in High Energy Materials (ACRHEM), University of Hyderabad, Prof. C. R. Rao Road, Gachibowli, \\ Hyderabad 500 046, Telangana, India, \\ ${ }^{5}$ School of Physics, University of Hyderabad, Prof. C. R. Rao Road, Gachibowli, Hyderabad 500 046, Telangana, India.
}

bmul2806@uni.sydney.edu.au

The development of carbon-neutral energy-generation is critical to combatting climate change. One such technology is the development of next-generation ion conductors for solid-oxide fuel cells (SOFCs). SOFCs offer a much more efficient method to extract energy from hydrogen or hydrocarbon fuels than current combustion engines due to their one-step chemical process. However, a bottleneck to the large-scale uptake of SOFCs is the poor performance of the conducting electrolytes that separate the anode from the cathode. Various lanthanoid fergusonite structures $\left(\operatorname{LnBO} \mathrm{O}_{4}\right)$ have recently been proposed as solid electrolyte candidates in solidoxide fuel cells, with increased high-temperature ionic conductivity being measured in chemically doped lanthanum orthoniobates $\left(\mathrm{LaNbO}_{4}\right)[1]$. However, a phase transition from $I 2 / a$ to $I 4_{1} / a$ within the operational temperature of SOFCs makes these structures nonideal.

To understand the effects of chemical doping on the structure and electrochemical properties of these fergusonite structures, several complex fergusonites have been investigated [2-3]. Of interest is the substitution of $\mathrm{Nb}^{\mathrm{V}}$ for $\mathrm{Ta}^{\mathrm{V}}$ on the $B$-site, which has shown a decrease in the unit cell volume of the structure [4]. This is particularly remarkable, given the two metal cations have the same ionic radius and Ta has an extra $5 d$ valence shell compared to the $4 d$ shell of $\mathrm{Nb}$. Such substitution has further shown to increase the $I 2 / a$ to $I 4_{1} / a$ first-order phase transition temperature, highlighting the potential of the properties of these structures to be specifically 'tailored' to be used for SOFCs.

Various solid-solution series of $\operatorname{Ln}\left(\mathrm{Nb}_{1-x} \mathrm{Ta}_{x}\right) \mathrm{O}_{4}(\operatorname{Ln}=\mathrm{La}-\mathrm{Lu})$ have been synthesised using conventional solid-state methods. Synchrotron X-ray and neutron powder diffraction methods have been used to investigate their structures, focusing on changes in both their unit cell volumes and the temperature of the $I 2 / a$ to $I 4_{1} / a$ phase transitions. Whilst the fergusonite structure is a monoclinic structure derived of the tetragonal scheelite aristotype, its structure is based on $B \mathrm{O}_{6}$ polyhedra as opposed to $B \mathrm{O}_{4}$ scheelite polyhedra. These studies have revealed several anomalies, revealing that different structures can be isolated by controlling the size of the $L n$ ion and synthetic conditions, and that the volume of the $\mathrm{BO}_{6}$ polyhedra and length of the $B-\mathrm{O}$ bonds change depending on its surrounding $L n$ ion. This data surprisingly implies that the $A \mathrm{O}_{8}$ polyhedra act as a rigid framework in which the $B \mathrm{O}_{6}$ polyhedra respond. The experimental data has been further reinforced by ground state energy calculations performed using density functional theory. This is a landmark accomplishment that has not been previously used in similarly studied structures. These insights can be used in the development and engineering of novel and advanced electrolyte materials for SOFCs.

[1] - Cao, Y.; Duan, N.; Yan, D.; Chi, B.; Pu, J.; Jian, L.; Enhanced Electrical Conductivity of LaNbO4 by A-Site Substitution. Int. J. Hydrogen Energy, 2016, 41 (45), 20633-20639.

[2] - Arulnesan, S. W.; Kayser, P.; Kimpton, J. A.; Kennedy, B. J.; Studies of the Fergusonite to Scheelite Phase Transition in LnNbO 4 Orthoniobates. J. Solid State Chem., 2019, 277, 229-239.

[3] - Ivanova, M.; Ricote, S.; Meulenberg, W. A.; Haugsrud, R.; Ziegner, M.; Effects of A- and B-Site (Co-)Acceptor Doping on the Structure and Proton Conductivity of $\mathrm{LaNbO}_{4}$. Solid State Ionics, 2012, 213, 45-52.

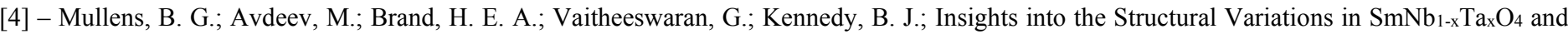
$\mathrm{HoNb}_{1-\mathrm{x}} \mathrm{O}_{4}$ Combined Experimental and Computational Studies. Dalton Trans., 2021, 50, 9103-9117.

Keywords: Disorder, Fuel Cells, Density Functional Theory, Fergusonite, Phase Transitions. 